\title{
LA DEVINETTE: \\ ÉTUDE LINGUISTIQUE ET DIMENSION CULTURELLE LE \\ CAS DE LA TSANJI:NA TUNISIENNE ${ }^{1}$
}

\section{THE DEVINETTE: \\ LINGUISTIC STUDY AND CULTURAL DIMENSION THE CASE OF TJANJI: NA TUNISIAN}

\author{
Leila Hosni2 \\ Université de Tunis 1
}

Resumo: $\mathrm{O}$ artigo trata do estudo de um tipo particular de discurso que não pode ser analisado independentemente de sua dimensão cultural, a saber, o tunisiano t:anfi: na (o enigma). Dessa forma, realiza uma descrição linguística que contempla a dimensão cultural.

Palavras-Chave: Adivinha; Cultura; Inferência; Tradução.

Abstract: We will study a particular type of discourse that cannot be studied independently of its cultural dimension: the tunisian tfanfi:na (the riddle). We will perform a linguistic description that will lead us to account for its cultural dimension.

Keywords: Guess what; Culture; Inference; Translation

Résumé: Il s'agit d'étudier un type particulier de discours qui ne peut être étudié indépendamment de sa dimension culturelle, à savoir la t fanfi:na tunisienne (la devinette). Nous en effectuerons une description linguistique qui nous mènera à rendre compte de sa dimension culturelle.

Mots-clés: Devinette; culture; inférence; traduction.

Article préparé en tant que produit du Projet Capes Cofecub 838/15.

2 Adresse e-mail de l'auteur: hosni_leila@yahoo.fr.

198 Nº60, Núm. Esp.|2018, Salvador: pp. 198-215 


\section{INTRODUCTION}

Malgré son importance dans l'étude des différents phénomènes linguistiques, la dimension culturelle demeure négligée. Les linguistes semblent la confier aux sociologues et aux anthropologues. De ce fait, nous trouvons, pour un même phénomène, une étude exclusivement linguistique (syntaxique, sémantique, etc.) et une étude qui ne s'intéresse qu'à sa dimension culturelle (les traditions, la religion, etc.). Cette dimension culturelle aurait toutefois dû "compléter" la description linguistique, étant donné que "langue et culture sont deux entités inséparables (...)" (MEJRI, 2016, p. 29) dont l'association est pertinente, voire indispensable dans l'étude de plusieurs phénomènes, surtout ceux dont l'encodage et le décodage sont effectués sur des bases typiquement culturelles. Nous pensons dans ce cadre aux "slogans", aux "énoncés parémiques", aux "contes populaires", etc.

Pour mettre en relief l'importance de la relation "linguistique"/ "culturel", nous nous proposons d'étudier un type d'énoncé qui témoigne de la "soudure" Langue/Culture dans la description linguistique, à savoir "la devinette", notamment la "tfanfi:na" tunisienne, une devinette populaire dont la description linguistique est étroitement liée à l'aspect culturel.

Après une brève définition de la "devinette" et une énumération de ses différents types, nous effectuerons une étude linguistique (formelle, syntaxique et sémantique) de la t fanfi:na tunisienne pour en arriver à l'importance de la dimension culturelle dans le traitement de ce type d'énoncé, ancré dans la culture populaire tunisienne.

\section{LA DEVINETTE: DÉFINITION ET TYPOLOGIE}

\subsection{Définition}

En définissant la devinette, les lexicographes, les linguistes, etc. évoquent sa dimension "ludique" et, par conséquent, "distractive"/"divertissante". En effet, le PR la définit comme un "jeu où l'on pose des questions", le TLFI, comme une "question formulée à quelqu'un dans le cadre d'un jeu d'esprit, pour qu'il devine la solution qu'elle requiert".

Les linguistes considèrent que, "grâce au biais ludique, la devinette établit dans un premier temps un contact social (...)" (ATMANI, 2011), que "les devinettes représentent un moment de distraction auquel s'adonnent à 
l'occasion la plupart des jeunes, garçons et filles" (ROULON-DOKO, 2004) et que la devinette est un “jeu enfantin (...)” (JACQUOT, 1981, p. 220).

Elle est également définie comme un énoncé oral. D'ailleurs, selon Jacquot (1981, p. 220), "la devinette pourrait [...] apparaître comme un genre mineur de la tradition orale $(. . .)^{\prime \prime}$. Elle peut, en effet, utilisée par les enfants à l'école :

Quand je suis sale je suis blanc et quand je suis propre je suis noir. Qui suis-je?

Comme elle peut faire l'objet des "soirées longues d'hiver » dans certaines sociétés, elle permet, de ce fait, de réunir la famille, et parfois les proches et les voisins. Elle consiste à poser des questions (l'énoncé de la devinette) pour lesquelles les participants au jeu doivent trouver des réponses. Généralement, ce sont les personnes les plus âgées (les plus sages) qui lancent la devinette. C'est dans ce sens qu'on considère qu'elle est issue du patrimoine oral d'une société ou d'une nation. Elle est même considérée, tout comme le conte, comme un type particulier de "la littérature populaire".

$\mathrm{Vu}$ sa "richesse" linguistique et culturelle, ce type d'énoncés a fait l'objet de plusieurs travaux de linguistique. Les uns ont valorisé la dimension culturelle et les autres l'ont négligée sans pour autant l'ignorer, puisqu'elle demeure un trait définitoire de la "devinette". Nous en citons:

Énigme et Devinette. Discussion (BENVENISTE, 1977)

Études. Beembe. (CONGO). Esquisse linguistique. Devinettes et proverbes (JACQUOT, 1981)

Les devinettes en pays gbaya (ROULON-DOKO, 2004)

La devinette: une pratique sociale. Étude linguistique. Corpus recueilli à Oujda (Maroc) (ATMANI, 2011).

Toutefois, l'appellation "devinette" ne désigne pas un seul type d'énoncé. On en distingue plusieurs types. Chaque type est identifié selon sa structure syntaxique, son contenu sémantique, son énonciateur, etc.

\subsection{Une typologie des devinettes}

\subsubsection{La charade}

C'est une "énigme où l'on doit deviner un mot de plusieurs syllabes décomposé en parties correspondant à un mot défini" (P.R):

Mon premier est entre 1 et 3.

Mon deuxième est l'inverse de la mort. 
Mon troisième est l'inverse de flou.

Que suis-je?

La solution: La devinette (deux, vie, net)

\subsubsection{Le logogriphe}

Il s'agit d'une "énigme où l'on donne à deviner plusieurs mots formés des mêmes lettres" (Ibid). Dans le mot «orange », par exemple, on trouve (par réduction et transposition): Oran, or, ange, orge, an, etc.

\subsubsection{La devinette frustrante}

Benveniste la définit comme une devinette caractérisée par l'ajout d'un "élément syntaxique non essentiel au fonctionnement du constituant dans lequel il se trouve" (1977, p. 80). Elle cite à titre d'exemple la devinette suivante:

Pourquoi Napoléon portait-il des bretelles tricolores?

où l'adjectif "tricolore" est un "ajout" qui désoriente le participant au jeu, la solution étant "pour tenir son pantalon".

\subsubsection{L'énigme}

C'est une "chose à deviner d'après une définition ou une description faite à dessein en termes obscurs, ambigus" (Ibidem). Elle est également appelée "devinette d'esprit" et considérée par E. Van Avermaet comme "de vraies devinettes, les devinettes d'intelligence où il faut trouver le mot de l'énigme"3 :

Aérienne et légère

Elle s'envole, éphémère.

Expédiée du Vatican,

Elle devient un commandement.

La solution: La bulle.

Dans ce travail, nous nous intéresserons à l'étude des "enigmes" ou tJanfi:na du dialecte tunisien. Nous tenterons de rendre compte de leur particularité linguistique en faisant intervenir la dimension culturelle.

\section{2 ÉTUDE LINGUISTIQUE DE LA TJANJI:NA TUNISIENNE}

Comme nous l'avons déjà mentionné, la t tanfi:na est un type particulier de devinette. Elle est énoncée en dialectal tunisien et proférée généralement par les personnes âgées qui la maitrisent ainsi que les réponses qui y réfèrent (cf.

${ }^{3}$ Cité dans C. Faïk-Nzuji Madiya (1976) 
infra). Sur le plan linguistique, elle se distingue des autres types de discours populaires, tels que "le conte" (l- $\chi$ ra:fa) en ce qu'elle est relativement courte, le proverbe, en ce qu'elle n'est pas toujours prosodique, etc. Cette différence réside également au niveau de sa structure. Il s'agit d'une "définition dialoguée" (TODOROV, 1973) ou, en d'autres termes, d'un discours bipartite constitué de "deux synonymes: une phrase et un mot isole" (ibid).

Étant un type particulier de discours populaire ou, selon certains autres dont Hassan Moubrak ${ }^{4}$ (2003), un genre particulier de "littérature populaire", la tfanfi:na présente des propriétés formelles, syntaxiques et sémantiques qui font sa particularité.

\subsection{Les propriétés formelles}

Sur le plan formel, la t tanfi:na tunisienne est essentiellement définie par sa musicalité et par la diversité de ses "formes" qui vont de la phrase simple au discours.

\subsubsection{La tJanfi:na: une structure prosodique?}

Elle présente, dans la majorité des cas, une structure prosodique, ce qui facilite la tâche du "questionneur" et lui permet de retenir ce type de devinette. On trouve cette prosodie, dans les devinettes qui présentent des structures binaires :

3bal fu:q 3bal // w 3bal jsaksak fer-rmal

Est-ce vraisemblable une montagne sur ne montagne et de la montagne s'écoule le sable? ${ }^{5}$

ternaires :

Sal-li re3lu Gu:d // w batnu Gu:d // w ra:su fi 3hannem mamdu:d//

Qui a le pied en bois, le ventre rempli d'air et la tête en enfer. et même quaternaire :

Sla anӨa mle:\{bija// a3mel men bne:t hle:1// kebret $\mathrm{w}$ wal-le:t sbija// w tzaw3et bu:ha zwe:3 hle:l.

C'est une belle fillette. Elle est plus belle que les filles de la tribune de Bni Hlel. Elle a grandi, est devenue jeune fille et a épousé son père d'une manière licite.

4 Un chercheur tunisien spécialisé dans le patrimoine et la littérature populaires.

5 La majorité des tfanfina du corpus sont traduites par Raouf Karray (2001). Certaines autres sont traduites par nous-même. Il s'agit dans ce cas, d'une traduction approximative, plus ou moins littérale (cf. infra) 
Ce qui rapproche la t tanji:na du genre poétique. Cette mélodie se manifeste également dans les allitérations qui consistent en une répétition des mêmes consonnes à l'intérieur de l'énoncé, ce qui est le cas de l'exemple suivant:

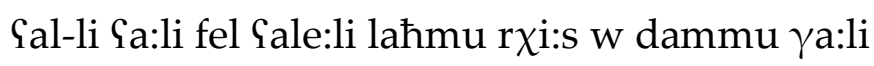

Qui est dans les hauteurs. Son sang est cher et sa viande n'a pas beaucoup de valeur,

où l'on assiste à la répétition de la consonne [९] et la rime [li].

Cependant, nous ne pouvons pas affirmer que les tfanfi:na tunisiennes sont toutes définies par cette dimension prosodique/rythmique. Notre corpus nous fournit également quelques tfanfi:na qui ne présentent aucun rythme. Il s'agit dans ce cas d'une ou d'un ensemble de phrases assertives ne présentant aucune prosodie, ce qui donne lieu, nous semble t-il, à des énoncés "lourds" difficile à retenir. Nous renvoyons, dans ce cas à l'exemple suivant:

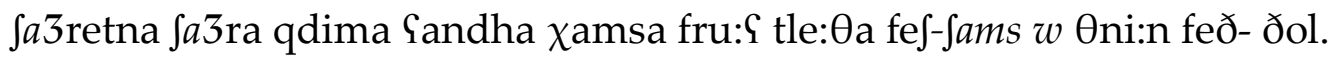

Notre vieil arbre a cinq branches, trois au soleil et deux à l'ombre.

Les tJanfi:na non prosodiques demeurent toutefois une exception. D'ailleurs, leur nombre dans le corpus est très réduit (2 sur 60), ce qui nous permet de définir ce type de discours populaire par sa prosodie. C'est ce qui facilite son apprentissage et permet de lui attribuer une dimension ludique.

\subsubsection{La t tanfi:na: de la phrase simple au discours}

La t tanfi:na peut figurer sous la forme:

- d'une phrase simple:

qad esjber jqi:m 3 bal

(celui qui a) la taille d'un empan porte une montagne

- d'une succession de phrases coordonnées:

Sal-li taSti ma:lha w tadhak w kol haj menha hje:tu

Celle qui distribue ses biens en riant, elle rend heureux tous les vivants

- de phrases subordonnées

Ke:nek qa:ri w fahhi:m w taqra hru:f lemde:res dilni ९la: Gu:d rawja:n nawartu $\mathrm{Su}$ :d je:bes

Si tu es compétent et lettré, dis-moi ce que c'est qu'une branche bien arrosée et sa fleur est une branche sèche.

- $\quad$ un discours (un texte)

Sla Өni:n mtla:zmi:n we:hid tfu:fu b९i:nek w ma tesmaSlu ani:n w

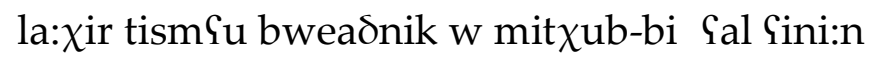


Deux choses liées. L'une est vue sans être entendue. L'autre est entendue sans être vue.

Qu'elle soit une phrase simple, une phrase complexe ou un texte, la tfanji:na figure toujours sous la forme d'une assertion et ce, malgré sa nature énigmatique. En d'autres termes, la principale particularité syntaxique de ce type de devinettes est qu'elle ne peut en aucun cas être une phrase interrogative.

\subsection{Les propriétés syntaxiques}

Excepté quelques cas, la tfanfi:na tunisienne figure dans un ensemble de moules syntaxiques, des moules qu'on ne trouve que dans le cadre de ce type de devinettes, ce qui nous mène à les considérer comme des "tSanfi:na prototypiques" :

De moule syntaxique: "Sla / Gal-li" + le contenu de la t tanfi:na

C'est le moule le plus fréquent dans le corpus. Dans le premier cas (Cla + le contenu de la tfanfi:na), la structure nous rappelle les phrases existentielles. En effet, la tJanfi:na suivante:

Sla laћma fi m $\gamma$ a:r la tintan la te $\underline{\text { da:r }}$

est littéralement traduite par une phrase existentielle:

Il y a une viande dans une cave qui ne pourrit ni ne moisit

Quant au deuxième moule "Sal-li + le contenu de la tfanfi:na", il correspond en français à une relative (Celui/ Celle qui), où le pronom démonstratif renvoie à la solution de la devinette:

Gal-li t3i:b ar-rizq w thiz ar-izq tạti za:dha bkul ra:ћa w tamla lu3u:h farha

$\rightarrow$ Celle qui apporte les biens et ramène les biens, elle donne sa nourriture avec aisance et remplit les visages de joie.

$>$ Le moule syntaxique "Sabdis-smad qa:1" (Abdessamad' a dit) + le contenu de la tJanfina.

Si le proverbe, par exemple, est introduit par l'expression « comme on dit», pour référer à un locuteur indéfini (inconnu), la tfanji:na est, dans un grand nombre de cas, introduite par l'expression "abdis-smad qa:l" désigne la

6 Cf. infra 
personne qui a créé la devinette en question. Nous renvoyons à la tfanfi:na suivante:

Gabdis-smad qa:l kilma:t w qa:l tsan-ntu ja Shu:di b९i:ni fuft larnib ta:kil

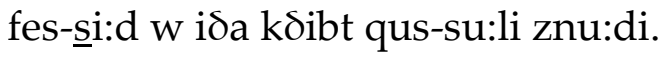

Abdessmad a dit des mots et a demandé aux témoins de l'écouter: "j'ai vu, de mes propres yeux, un lapin qui mange un lion, et si je mens, coupez-moi les bras".

Le moule syntaxique: ka:nik qa:ri $w$ fhi:m + Verbe de parole à l'impératif + le contenu de la tJanfina.

Il s'agit dans ce cas d'une phrase hypothétique introduite par la subordonnée « ka:nik qa:ri w fhi:m », littéralement traduite par "si tu es lettré et inteligente". Cette structure est souvent suivie d'un verbe de parole à l'impératif comme

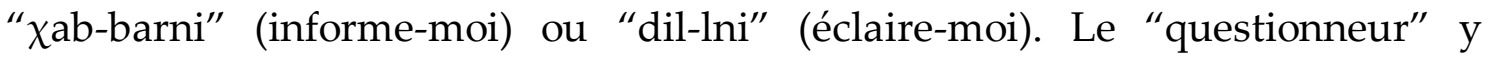
recourt pour demander, directement, une réponse au devineur:

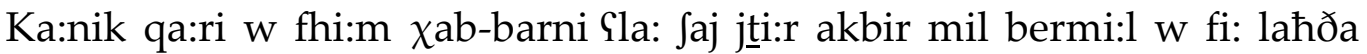

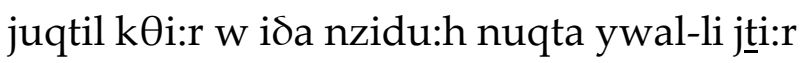

Si tu es lettré et intelligent, informe-moi sur quelque chose plus grand que le barrel et en un moment il tue plusieurs personnes, et si on lui ajoute un point, il vole.

Lorsqu'elles ne figurent pas dans ces différents moules syntaxiques, les tfanfi:na sont généralement des phrases dont le thème est l'élément auquel réfère la solution:

3bal fu:q 3bal w 3bal jsaksak fir-rmal

Est-ce vraisemblable une montagne sur une montagne et de la montagne s'écoule le sable?

Dans ce cas, les "montagens" superposées réfèrent aux deux parties superposées de "la meule de moulin", la solution de la devinette.

La diversité des structures syntaxiques de la tJanfi:na tunisienne témoigne de sa richesse linguistique, une richesse qui se manifeste également sur le plan sémantique.

\subsection{Les propriétés sémantiques}

Sur le plan sémantique, seront étudiés les thèmes sur lesquels portent les t fanfi:na. Il s'agit de vérifier si ces dernières portent seulement sur des thèmes « traditionnels », ou également sur des thèmes appartenant à la vie moderne. 
Aucune t fanfi:na du corpus ne réfère à "la vie moderne" du tunisien. Elles réfèrent toutes à la vie quotidienne d'un tunisien "traditionnel", citadin ou paysan. On distingue donc des thèmes qui renvoient à son corps, aux végétaux, aux métiers (artisanaux et agricoles), aux ustensiles ménagers, et surtout à la religion (l'Islam).

$>$ Le corps humain

Il s'agit surtout des parties du corps, comme le doigt / l'ongle et la langue, dans :

Ka:nik qa:ri w fah-hi:m w taqra hru:f limda:ris dil-lni ৎla: 乌u:d rawja:n nawartu Gu:d ja:bis

Si tu es lettré et intelligent, dis-moi ce que c'est qu'une branche bien arrosée et sa fleur est une branche sèche.

Sla laћma fi m $\gamma$ a:r la titin la te $\chi \underline{\text { da:r }}$

Il y a une viande dans une cave qui ne pourrit ni ne moisit

$>$ Les végétaux:

Ce sont essentiellement les légumes et les fruits produits en Tunisie comme les olives :

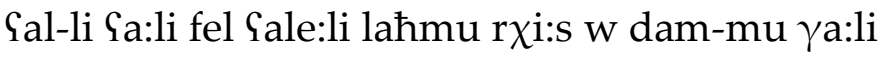

Il existe là haut. Son sang est cher et sa viande n'a pas beaucoup de valeur

$>$ Les métiers

Ces t tanfi:na portent surtout sur les outils "traditionnels" utilisés dans les différents métiers (agricoles et artisanaux) exercés par le Tunisien. Nous en citons par exemple la charrue, utilisée par l'agriculteur:

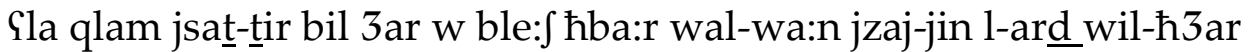

Quel est ce calame qui trace des lignes sans encre ni couleurs pourtant il orne la terre et les pierres?

et le soufflet du forgeron :

Sal-li ri3lu Gu:d // w batnu Gu:d // w ra:su fi 3han-nim mamdu:d//

Celui qui a le pied en bois, le ventre rempli d'air et la tête en enfer.

$>$ Les ustensiles ménagers

Les t tanfi:na tunisiennes réfèrent également aux équipements de la cuisine tunisienne. Ces équipements présentent tous des ustensiles traditionnels, tels que la meule, une sorte de moulin qui sert à moudre le blé (cf. supra) et le théière:

Sla Ja:jib dehri w-awla:du fla:lis huwa jạti lawla:du wa-wla:du tạti lenna:s. 
Il y a un vilain vieux monsieur dont les enfants sont pauvres. Il donne tout à ses enfants et ses enfants donnent aux gens.

$>$ La religion musulmane

Le Tunisien accorde beaucoup d'importance à la religion, qui intervient dans tous les domaines de sa vie (le mariage, l'héritage, etc.). Elle est, de ce fait, dominante dans la t tanfi:na qui peut, entre autres, porter sur la prière de vendredi :

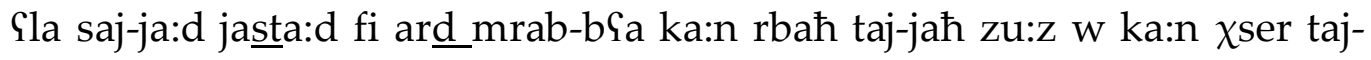
jah arbSa

Il y a un chasseur qui chasse dans une terre carrée. S'il chasse "deux", il est gagnant et s'il chasse "quatre", il est perdant.

et les fêtes religieuses (Aid El Fitr" et Aid El Edhha ${ }^{8}$ ) :

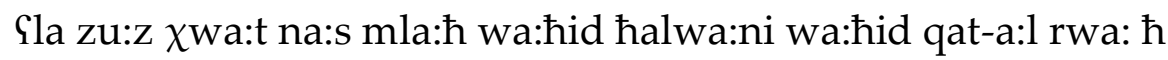

Il y a deux frères très gentils. L'un est pâtissier et l'autre est assassin.

La tJanfi:na n'a pas été actualisée dans la société tunisienne. C'est pour cette raison qu'on la voit disparaître de nos jours et que, même en y recourant, seuls les devineurs âgés, peuvent la résoudre. Cela est essentiellement dû à la dimension culturelle qui intervient dans l'interprétation de ce genre d'énoncés: le questionneur et le devineur doivent avoir un savoir partagé, ce savoir étant essentiellement culturel.

\section{POUR INTERPRÉTER LA TJANJI:NA: DE LA CULTURE AU LEXIQUE}

Faisant partie de la littérature populaire, la tfanfi:na s'intéresse à tous les domaines "traditionnels" de la vie du Tunisien. C'est dans ce sens que, comme nous l'avons déjà mentionné, toutes les solutions des t tanfi:na ne renvoient pas à la vie moderne. Par conséquent, leur interprétation a besoin d'un acquis culturel, c'est-à-dire d'une connaissance de la culture tunisienne, mais dans une époque bien déterminée.

L'importance de la dimension culturelle réside dans l'interprétation de ce type de devinettes, une interprétation qui nécessite un processus inférentiel basé aussi bien sur "une culture linguistique" (le lexique) que sur "une culture extra-linguistique" (les connaissances partagées). De ce fait, un devineur qui

\footnotetext{
C'est la fête qui marque la rupture du jeûne du mois de ramadan, chez les musulmans, où l'on consomme et distribue des confiseries.

8 C'est la «fête du sacrifice », chez les musulmans, où l'on égorge le mouton.
} 
ignore ces aspects culturels serait incapable de trouver la solution. Cette caractéristique rapproche ce type de devinettes des expressions idiomatiques.

Après un bref aperçu historique de la t tanfi:na tunisienne et une description de son état actuel, nous nous concentrerons sur l'importance de la dimension culturelle dans son interprétation et ce, en prouvant qu'il s'agit d'une séquence figée idiomatique, qui ne peut être interprétée que dans le cadre d'un environnement culturel partagé ; et en étudiant sa "traductibilité ", la traduction étant également une activité étroitement liée à la culture.

\subsection{Quel statut de t $\int a n \int i: n a$ tunisienne}

\subsubsection{Aperçu historique}

Tout comme le conte populaire (l- $\chi \mathrm{ra}: \mathrm{fa})$, la t tanfi:na faisait partie de la vie quotidienne du Tunisien. Elle fut proposée par les parents et les grandsparents aux enfants qui se concurrençaient pour la résoudre. Par conséquent, elle présentait un moyen de divertissement, mais aussi un jeu d'esprit qui permettait de développer les capacités intellectuelles des enfants. Un témoignage de Raouf Karray, l'auteur d'un ouvrage intitulé "Devinettes de Tunisie" (2001), permet d'ancrer la tJanfi:na tunisienne dans son contexte historique :

Tantôt nous jouions, tantôt nous nous réfugions près du tronc d'un arbre à l'ombre et aux fruits abondants. Nous répétions les histoires ou les devinettes que chacun d'entre nous avait retenues, après les avoir entendues par une froide nuit d'hiver, lorsque nous rentrions le soir à la maison, fatigués par une leçon ennuyeuse ou éreintés d'avoir longtemps joué. Nous nous emmitouflions avec hâte dans une couverture de laine et, blottis dans les bras de notre grand-mère ou entre ses jambes, nous écoutions quelques-unes de ses belles histoires ou nous rivalisions pour trouver la solution des devinettes qu'elle nous posait et qui, chaque fois, semblaient plus compliquées 9 .

Il semble que l'un des fondateurs de ce type d'énoncés serait Abdessamad Ecchebbi. Connu par sa sagesse et sa ruse, il a pu influencer la tribu qu'il dirigeait, entre autres, par "l'invention" de devinettes, ce qui a fait sa célébrité. D'ailleurs, son nom figure dans un grand nombre de t fanfi:na dont il est, nous semble t-il, "l'élaborateur" (cf. supra).

9 Un extrait d'un article publié dans la revue en ligne « Takam Tikou ». 


\subsubsection{Statut actuel de la tfanfi:na}

Avec l'évolution technologique, sociale et économique, la tfanfi:na a perdu son statut initial. Elle ne représente plus un moyen de divertissement et un jeu d'esprit destiné aux enfants. On y recourt rarement. Les deux partenaires du jeu sont généralement des adultes qui en sont nostalgiques. De nos jours, on lui substitue les alya:z, des énigmes, en arabe littéral, qu'on trouve généralement dans les manuels scolaires, destinés aux élèves du primaire et du collège et par "les devinettes frustrantes" (BENVENISTE), proférées par les jeunes tunisiens, en arabe dialectal. Leur objectif est essentiellement ludique, humoristique, etc. sans pour autant être un jeu d'esprit.

En rendant compte des contextes historique et actuel de la tJanfi:na tunisienne, nous sommes parvenue à conclure qu'il s'agit d'un genre littéraire dont la compréhension et l'interprétation sont relatives à "une époque" et par conséquent, tributaires d'une "culture" (une société, un patrimoine, un univers de croyances, etc.). Elles fonctionnent donc comme des expressions idiomatiques.

\subsection{La t $\int a n \int i: n a$ tunisienne : figement et idiomaticité}

Toutes les tfanfi:na tunisiennes sont des séquences figées. Elles partagent avec ces dernières, entre autres, "la polylexicalité", "le blocage des propriétés transformationnelles", "le blocage des paradigmes synonymiques", etc. Nous pouvons de ce fait les considérer comme "des expressions idiomatiques", dans la mesure où elles présentent une "forme spécifique que prend la substance (ou la matière) linguistique dans une langue donnée" (MEJRI, 2006, p. 144) et qu'elles se distinguent par le fait qu'elles sont spécifiques au dialecte tunisien et qu'elles résistent à la traduction. Leur caractère idiomatique soumet leur interprétation à des conditions d'ordre culturel. Pour inférer "la solution", le devineur doit maîtriser aussi bien le lexique du dialecte tunisien, notamment les relations lexicales, que les traditions, le patrimoine, l'histoire, etc. C'est pour cette raison que nous proposons de distinguer, dans l'interprétation des t Janfina, deux types de processus inférentiels : lexical et extra-linguistique.

a) L'inférence lexicale

La t fanfi:na tunisienne est une énigme (cf. supra.) qui, " sans nommer une chose, [...] la décrit par ses causes, ses effets et ses propriétés, mais sous des termes et des idées équivoques pour exciter l'esprit à la découvrir" (BERRA, 2008, p. 11). C'est le recours aux éléments linguistiques référant, entre autres, 
aux "causes", aux "effets" et aux "propriétés" qui permet d'interpréter la solution de ce type de devinette. Le devineur recourt donc au mécanisme d' "inférence lexical", un type d'inférence qui "permet de s'approcher du sens des mots inconnus à travers les indices linguistiques présents dans un texte" (HAASTRUP \& KINTSCH, 1991). La solution sera inférée à travers l'identification d'un ensemble de relations lexicales, dont des relations tropiques, établies entre un/ des éléments de la tfanfi:na et une éventuelle solution. Nous distinguons plusieurs types de relations:

Une relation hyperonymique :

Le premier mot de la tfanfi:na est un hyperonyme englobant l'unité lexicale à laquelle renvoie "la solution":

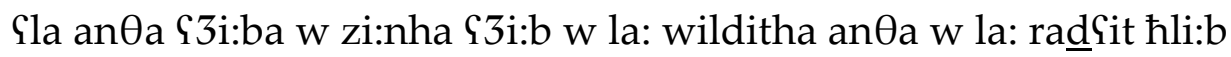

Il y a une femme étrange, ayant une beauté étrange. Elle n'a été ni engendrée ni allaitée,

où le $\mathrm{SN}$ femme est un terme générique qui englobe "Eve", la solution de la devinette.

\section{$>\quad$ Une relation métaphorique (comparant / comparé)}

L'un des composants de la tJanfi:na (généralement le premier élément) est une métaphore qui réfère à la solution, comme c'est le cas de montagne, dans l'exemple suivant:

3bal fu:q 3bal // w 3bal jsaksik fir-rmal

Est-ce vraisemblable une montagne sur ne montagne et de la montagne s'écoule le sable?

Dans certains cas, le recours à la métaphore contribue à la personnification de l'objet, du fruit, du légume, etc. auxquels réfère la solution, ce qui accentue la devinette et augmente l'effort interprétatif du devineur. Nous pensons, entre autres, à la devinette suivante:

Sla fa:jib dihri w-awla:du fla:lis huwa jạti lawla:du wa-wla:du tạti linna:s.

Il y a un vilain vieux monsieur dont les enfants sont pauvres. Il donne tout à ses enfants et ses enfants donnent aux gens.

où vieux monsieur réfère à la "théière".

Dans d'autres cas, nous assistons à la démarche inverse, dans la mesure où on infère un être humain en le chosifiant ou en l'animalisant. "Une femme qui ne jeûne pas au mois de Ramadhan pour des raisons légitimes" est par exemple comparée à "un lapin": 
Sabdes-smad qa:l kilma:t w qa:l tsan-ntu ja Shu:di bCi:ni fuft larnib ta:kil

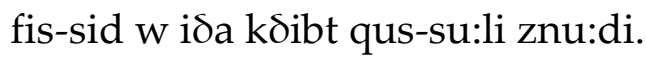

Abdessmad a dit des mots et a demandé aux témoins de l'écouter: "J'ai vu, avec mes propres yeux, un lapin qui mange un lion, et si je mens coupez-moi les bras".

$>\quad$ Une relation méronymique

Dans cet exemple:

Sal-li ri3lu Gu:d // w batnu Gu:d // w ra:su fi 3han-nim mamdu:d//

Celui qui a le pied en bois, le ventre rempli d'air et la tête en enfer.

on a inféré un holonyme "le soufflet du forgeron" grâce à ses méronymes "pied", "ventre" et "tête" qui véhiculent, ici, un sens figuré (métaphorique), ce qui complique l'interprétation de la devinette.

Ces différents types de relations lexicales sont établis entre "la solution" inférée et l'un ou quelques éléments linguistiques de la t fanfina. Le devineur ne peut toutefois pas se contenter de ces inférences lexicales. Il doit les appcroiser avec d'autres connaissances partagées (extra-linguistiques.). Un autre processus inférentiel intervient donc pour compléter le premier et pour, finalement, trouver la solution.

\subsubsection{L'inférence extra-linguistique}

Nous considérons que l'inférence lexicale est un «simple» point de départ dans l'interprétation des t tanfi:na tunisiennes. Ces dernières sont également interprétées par le biais du processus de l' "inférence extralinguistique". Le devineur fera également intervenir son acquis culturel, basé sur des connaissances:

\section{$>$ Religieuses}

Dans la devinette suivante:

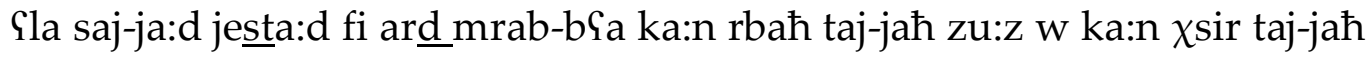
arbৎa

Il y a un chasseur qui chasse dans une terre carrée. S'il chasse "deux", il est gagnant et s'il chasse "quatre ", il est perdant,

seul le devineur qui maîtrise "les règles" de la prière peut comprendre que: le "gagnant" est celui qui fait la prière du Vendredi à la mosquée (deux rakaâ) et le "perdant" est celui qui prie à la maison (quatre rakaâ). La solution est par conséquent "la prière du Vendredi". 
$>$ Culinaires:

Pour trouver la solution de cette tfanfi:na (La meule du moulin) :

3bal fu:q 3bal w 3bal jsaksik fir-rmal

Est-ce vraisemblable qu'une montagne sur une montagne et de la montagne

s'écoule le sable?

il faut déjà connaître la forme de cet ustensile de cuisine (deux pierres superposées) et son fonctionnement (les céréales se transforment en une poudre qui ressemble au sable).

$>$ Patrimoniaux

L'interprétation $\mathrm{du}$ « tatouage » comme solution de la t fanfi:na suivante:

Ka:nik qa:ri w fhi:m w tefhim hru:f l-mutnab-bi fi:k ha:3a mif $\chi$ li:qit rabbi

Si tu es lettré et intelligent, tu comprendras qu'il y a en toi quelque chose d'artificiel,

nécessite une connaissance du patrimoine tunisien. Le tatouage représente en effet une tradition berbère. Il concerne aussi bien les hommes que les femmes. Son objectif est essentiellement esthétique.

Pour inférer la solution d'une tfanfina, il ne suffit pas de chercher les inférences lexicales entre cette dernière et sa solution. Il faut également faire appel aux connaissances "culturelles" partagées. C'est l'association de ces deux processus inférentiels qui permet de résoudre ce type de devinette, ce qui confirme son caractère idiomatique. Cette idiomaticité contribue aussi au blocage de la traduction de ce type d'énoncé.

\subsection{La $t \int a n \int i: n a$ tunisienne: une traduction de la culture?}

“(...) le passage d'un code linguistique à un autre est un phénomène complexe, puisqu'il implique plusieurs paramètres non seulement linguistiques, mais aussi bien pragmatiques que culturels" (BACCOUCHE, 2003, p. 96). Comme tous les types d'énoncés (littéraires, religieux, etc.), la traduction des tfanfi:na est soumise à cet ensemble de contraintes "pragmatiques" et "culturelles". Ces contraintes sont consolidées par son caractère idiomatique. Nous tenterons d'expliquer cette idée à travers la traduction de la tJanfi:na tunisienne déjà effectuée par Raouf Karray, dans son ouvrage Devinettes de Tunisie (2001), un livre destiné aux enfants tunisiens (arabophones et francophones). Il présente un ensemble de devinettes 
tunisiennes, traduites en français. Les solutions sont également traduites, et illustrées par des images.

La traduction de ces tfanfi:na montre qu'il est possible de traduire " les expressions idiomatiques », des expressions par définition intraduisibles ${ }^{10}$. La devinette suivante :

qața min Pand il had-da:d qat?a min Pand il 3az-za:r w qat?a min Pand in-na3-3a:r wil-li bi:n jdi:k tar,

est traduite par:

Le forgeron m'a donné un morceau, le boucher m'a donné un morceau, le menuisier m'a donné un morceau. Avec ma drôle de tête je vais faire la fête.

Cette traduction n'est toutefois pas "fidèle"dans la mesure où elle donne lieu à "une rupture", tantôt formelle tantôt syntaxique tantôt sémantique par rapport à l'énoncé de départ.

\subsubsection{Une rupture formelle}

En traduisant la devinette suivante:

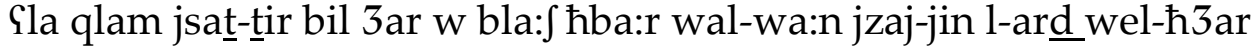

$\rightarrow$ Quel est ce calame qui trace des lignes sans encre ni couleurs pourtant il orne la terre et les pierres?

Le traducteur trahit sa forme initiale. Il s'agit dans ce cas d'un passage de la forme assertive à la forme interrogative. Pourtant, comme nous l'avons déjà mentionné, la t fanfi:na tunisienne ne figure que sous la forme d'une assertion (cf. supra).

\subsubsection{Une rupture syntaxique}

En énonçant sa devinette, le questionneur s'attend à "une solution". C'est dans ce sens que nous assimilons la tJanfi:na à une interrogation partielle. Mais, la traduction de cette t fanfi:na:

3bal fu:q 3bal w 3bal jsaksik fir-rmal

$\rightarrow$ Est-ce vraisemblable une montagne sur une montagne et de la montagne s'écoule le sable?

10 Nous avons eu beaucoup de difficultés à traduire nous-même les tfanfina du corpus, qui n'existent pas dans l'ouvrage Devinettes de Tunisie, ce qui nous a poussée, dans la majorité des cas, à recourir à une traduction littérale. 
a transgressé la "règle": on recourt plutôt à une interrogation totale introduite par "est-ce vraisemblable", une question qui nécessite une réponse par "oui" ou "non".

\subsubsection{Une rupture sémantique}

Dans les exemples précédents, le traducteur a marqué une rupture formelle et syntaxique avec l'énoncé initial. Mais il en a gardé le contenu sémantique, ce qui nous rapproche d'une traduction littérale. En revanche, la traduction de la devinette suivante:

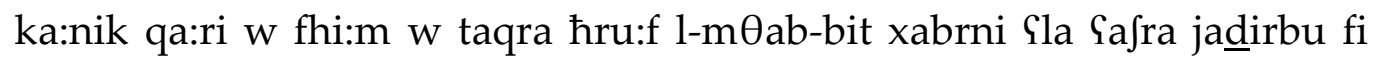
mej-jit,

qui peut être littéralement traduite par:

Si tu es lettré et intelligent informe-moi sur Dix qui battent un mort, donne lieu à une rupture sémantique. Le passage de L1 à L2 est accompagné d'un changement du sens de la devinette:

Dix petits bonhommes ont trouvé du bois mort, lui ont gratté le ventre, chatouillé le cou et il s'est mis à chanter

Le traducteur a donc "inventé" une autre devinette, véhiculant un contenu sémantique différent pour faciliter la tâche du devineur francophone, la devinette tunisienne étant brève et moins développée.

Dans ces trois cas de figure, nous n'avons pas affaire à "une traduction" de la tfanfi:na tunisienne. Il nous semble que l'objectif de l'auteur de l'ouvrage ne consiste pas à traduire ces devinettes, mais à trouver des devinettes plus ou moins équivalentes tout en gardant un aspect définitoire de ce type d'énoncés, à savoir "le rythme" et "la musicalité".

\section{CONCLUSION}

Bien qu'elle soit en voie de disparition, la t tanfi:na tunisienne demeure, comme tous les autres types de "discours populaires", l'un des piliers de la culture tunisienne. Outre sa fonction ludique, elle rend compte de la culture, du patrimoine et de l'histoire d'un peuple. Sa richesse culturelle est renforcée par une densité linguistique. Elle présente, en effet, un genre particulier de "la littérature populaire", ayant ses propres caractéristiques et son propre fonctionnement linguistique (formel, syntaxique et sémantique).

Une étude exhaustive de ce type d'énoncés, que nous considérons comme des expressions idiomatiques, nécessite, par conséquent, le recours 
aussi bien au volet linguistique qu'au volet culturel. Cette idée se confirme lors de la traduction, une activité où l'on ne traduit pas seulement le lexique, mais aussi "la culture".

\section{RÉFÉRENCES}

ATMANI, Latifa. La devinette: une pratique sociale. Étude linguistique. Corpus recueilli à Oujda (Maroc). 2011. 538 f. Thèse. (Doctorat en linguistique) Université Paris V, Paris.

BENVENISTE, Blanche C. (1977), Énigme et Devinette. Discussion. In: Bulletin de l'Association d'étude sur l'humanisme, la réforme et la renaissance, 1977, n.7. Actes du colloque de Goutelas. Paris: Facétie et littérature facétieuse à l'époque de la Renaissance, 29 septembre-1er octobre 1977, p. 79-91.

BERRA, A. Théorie et pratique de l'énigme en Grèce ancienne. In: Matringe, Denis Études classiques. Paris: Ecole des Hautes Etudes en Sciences Sociales (EHESS), 2008. p. $10-23$.

FAÏK-NZUJI MADIYA, C. Devinettes tonales Luba et Luluwa Tsumwinu. Paris: SELAF, 1976.

HAASTRUP, Kintsch W. Lexical inferencing procedures or talking about words. Tubingen: Gunter Narr, 1991.

JACQUOT, A. Etudes BEEMB (Congo): Esquisse linguistique Devinettes et Proverbes. Paris: O.R.S.T.O.M, 1981.

KARRAY, R. Devinettes de Tunisie. Nimes: Grandir, 2001.

MEJRI, S. Les mot du jeu: entre idiomaticité et culture. In: Dotoli, Giovanni; Mejri, Salah. Les cahiers du dictionnaire $\mathrm{N}^{\circ}$ 8, Les mots de la Méditerranée dans le dictionnaire. Paris: Classiques Garnier, 2017, p. 29-46.

MEJRI, S. La terminologie du figement: approche contrastive (français-Arabe). In: NEVEU, Franck. Syntaxe et sémantique n. 7, La terminologie linguistique. Problèmes épistémologiques, conceptuels et traductionnels. Caen: Presses Universitaires de Caen, 2006, p. 139-152.

ROULON-DOKO, P. Les devinettes en pays gbaya. P. Boyeldieu et P. Nougayrol. In: BOYELDIEU, P. et NOUGAYROL, P. Langues et cultures: terrains d'Afrique, Hommage à F. Cloarec-Heiss. Peteers: Afrique et langage 7, p.121-135, 2004.

Nota do editor:

Artigo submetido para avaliação em: 06 de dezembro de 2018.

Aprovado em sistema duplo cego em: 18 de dezembro de 2018. 